\title{
Expression of miR-129-5p and miR-433 in the serum of breast cancer patients and their relationship with clinicopathological features
}

\author{
JINHUI XUE ${ }^{1 *}$, XIAOHAN ZHU ${ }^{2 *}$, PEI HUANG ${ }^{3}$, YINGYING HE $^{1}$, \\ YANJING XIAO $^{1}$, RUIHAN LIU $^{1}$ and MIN ZHAO ${ }^{4}$
}

\begin{abstract}
${ }^{1}$ Department of Pathology, Zhengzhou Central Hospital Affiliated to Zhengzhou University, Zhengzhou, Henan 450007; ${ }^{2}$ College of Nursing, Zheng Zhou Railway Vocational and Technical College, Zhengzhou, Henan 451460; ${ }^{3}$ Department of Pathology, The First Affiliated Hospital of Zhengzhou University, Zhengzhou, Henan 450052; ${ }^{4}$ Department of Neurology, Zhengzhou Central Hospital Affiliated to Zhengzhou University, Zhengzhou, Henan 450007, P.R. China
\end{abstract}

Received January 21, 2020; Accepted May 6, 2020

DOI: $10.3892 / \mathrm{ol} .2020 .11827$

\begin{abstract}
Expression of miR-129-5p and miR-433 was detected in breast cancer to explore the relationship with clinicopathological features of breast cancer. Seventy-eight patients with breast cancer diagnosed in Zhengzhou Central Hospital Affiliated to Zhengzhou University (Zhengzhou, China) from February 2016 to September 2017 were collected and enrolled into the research group. Additionally, 72 healthy people who underwent physical examination during the same period were selected as the control group. The expression levels of miR-129-5p and miR-433 in peripheral blood of the two groups were detected by fluorescence quantitative PCR (RT-PCR). The relationship between the expression of miR-129-5p, miR-433 and clinicopathological features, clinical stages of breast cancer, and the degree of differentiation were analyzed. Expression of miR-129-5p and miR-433 in the research group was significantly lower than that in the control group $(\mathrm{P}<0.05)$. Expression of miR-129-5p in the blood of breast cancer patients was correlated with tumor size, differentiation degree, lymph node metastasis, depth of invasion and clinical stages $(\mathrm{P}<0.05)$. Expression level of miR-433 was correlated with the degree of differentiation, lymph node metastasis, depth of invasion and clinical stages $(\mathrm{P}<0.05)$. miR-129-5p and miR-433 were positively correlated with differentiation degree
\end{abstract}

Correspondence to: Dr Min Zhao, Department of Neurology, Zhengzhou Central Hospital Affiliated to Zhengzhou University, 16 Tongbai North Road, Zhengzhou, Henan 450007, P.R. China

E-mail: bze2ge@163.com

*Contributed equally

Key words: miR-129-5p, miR-433, breast cancer, clinicopathological features $(\mathrm{r}=0.8507, \mathrm{r}=0.7522 ; \mathrm{P}<0.05)$, and negatively correlated with clinical stages $(r=-0.6595,-0.8947 ; \mathrm{P}<0.05)$. The sensitivity and area under curve (AUC) were higher in joint detection (87.5 and $0.95 \%$ respectively), compared with those in single detection. Patients were separated into high and low expression groups according to the median values of miR-129-5p and miR-433. The one-year survival rate of breast cancer patients was analyzed. Patients in the low expression groups had lower survival rates than patients in the high expression groups $(\mathrm{P}<0.05)$. In conclusion, the expression of miR-129-5p and miR-433 in peripheral blood of breast cancer patients is lower than that of healthy people, and the expression level is closely related to clinical stages and differentiation degree, which is expected to provide reference value for judging the state of breast cancer patients. The combined detection of miR-129-5p and miR-433 is of great significance in the diagnosis and treatment of breast cancer.

\section{Introduction}

Cancer, one of the top ten malignant diseases, is a severe threat to all human beings (1). World Health Organization (WHO) and International Agency for Research on Cancer (IARC) concluded that there were 14.09 million new cases of malignant tumors such as breast, lung and colorectal cancer, and 8.2 million deaths in 2012 (2). Approximately 1.2 million women are diagnosed with breast cancer every year in the world, and about 500,000 women die of breast cancer every year (3). In 2012, in China there were about 190,000 women diagnosed with breast cancer, ranking first in the incidence of female diseases, and 50,000 deaths, ranking sixth. It is the most common with the highest incidence malignant tumors among women in the world (4), and the second cause of death of female cancer in the world (5). Moreover, in recent years, the incidence of breast cancer is still rising $(6,7)$ and the proportion of the incidence and death of all malignant tumors in women worldwide is also increasing $(8,9)$. Although China has a low incidence of breast cancer, the burden of breast cancer 
in China is still increasing (10). At present, the sensitivity and specificity of clinical markers, such as CEA and CA19-9, are not high. In recent years, many studies have shown the value of serum miRNA in tumor diagnosis (11).

miRNA is involved in the regulation of various biological behaviors and has been found to be a target for diagnosis and treatment of various diseases (12). A previous study pointed out that miRNA can be used for disease diagnosis, treatment monitoring and predicting prognosis (13). MicroRNAs (miRNAs) are found in many organisms such as animals, plants and viruses. They are a class of endogenous non-coding small RNAs (14) with a length of about $22 \mathrm{nt}$. It has been shown in literature that miRNAs can be involved as protooncogenes or tumor suppressor genes in the development and progression of tumors $(15,16)$. Studies have also found that miRNAs can participate in the regulation of their downstream target genes in the case of multidrug resistance (MDR) $(17,18)$. As tumor-associated miRNAs in many cancers such as breast, gastric, bladder, ovarian, liver cancer and oral squamous cell carcinoma, miR-129-5p (19-21) and miR-433 $(22,23)$ can affect proliferation, invasion, migration and apoptosis of many cancer cells, and can also regulate chemotherapeutic resistance. Studies have shown the mechanism of action of miR-433 and miR-129-5p in breast cancer $(24,25)$, but miR-433 and miR-129-5p have rarely been studied in the diagnosis of breast cancer.

In the present study, the expression of miR-129-5p and miR-433 in breast cancer was detected to study the relationship between the expression levels of miR-129-5p, miR-433 and clinicopathological characteristics, and to explore the relationship between the expression levels of miR-129-5p and miR-433 in the blood of breast cancer patients with different clinical stages and differentiation. The values of single and combined diagnosis of miR-129-5p and miR-433 in breast cancer were compared to provide a theoretical basis for early diagnosis and treatment of breast cancer.

\section{Patients and methods}

General information. Seventy-eight patients with breast cancer diagnosed in Zhengzhou Central Hospital Affiliated to Zhengzhou University (Zhengzhou, China) from February 2016 to September 2017 were enrolled in the research group, aged $58.52 \pm 7.96$ years. There were 16 cases highly differentiated, 45 cases moderately differentiated and 17 cases poorly differentiated. Clinical stages, 16 cases in stage I, 25 cases in stage II, 21 cases in stage III and 16 cases in stage IV. Seventy-two healthy people who received physical examination in the same period were included in the control group, aged $57.98 \pm 8.57$ years.

Inclusion criteria: Patients with complete clinical and pathological data; patients without neoadjuvant chemotherapy, radiotherapy and immunotherapy; patients who received three routine examinations, liver and kidney function, electrocardiogram and other routine examinations; patients who were diagnosed with breast cancer by postoperative pathological reports; patients who were accompanied by family members at the time of admission.

Exclusion criteria: Patients with history of mental illness and family history of mental illness; patients with autoimmune deficiency; patients with history of severe organ disease, craniocerebral trauma, drug dependence; patients with communication disorder due to aphasia, irritability, unconsciousness and other factors and cannot cooperate with the examiner.

This study was approved by the Ethics Committee of the Zhengzhou Central Hospital Affiliated to Zhengzhou University. The patients and their families were given a detailed description of the experimental procedures prior to the study, and a complete informed consent was signed by the patients and/or guardians.

Blood collection. A total of $4 \mathrm{ml}$ peripheral blood on an empty stomach was collected in the morning from patients in the research group and put into an anticoagulant tube. Fasting peripheral blood $(4 \mathrm{ml})$ was also collected from the controls in the morning on the day of physical examination and was put into an anticoagulant tube. After coagulation for $60 \mathrm{~min}$ at $20-25^{\circ} \mathrm{C}$, it was centrifuged at $1,006.2 \times \mathrm{g}$ for $10 \mathrm{~min}$, with a radius of $10 \mathrm{~cm}$ at $4{ }^{\circ} \mathrm{C}$, the upper serum was separated for storage, avoiding repeated freezing and thawing.

Laboratory instruments and agents. TRIzol kit (Shanghai Mingjing Biology Co., Ltd.; 5003050), DNase 1 (Shanghai Hengfeng Biotechnology Co., Ltd.; K003399P), cDNA reverse transcription kit (Shanghai Jianqiao Technology Co., Ltd.; 4368814), ultraviolet spectrophotometer (Shanghai Hengfei Biotechnology Co., Ltd.; UV-1100), fluorescence quantitative PCR kit (Beijing Baioleibo Technology Co., Ltd.; ALH190-UBN), and real-time PCR detector (Agilent Technologies Co. Ltd.; MX3000P).

Fluorescence quantitative polymerase chain reaction (RT-PCR) experimental procedures. Total RNA in serum was extracted in accordance with the instructions of TRIzol kit. Template RNA was digested by DNase I (RNA free) to eliminate genomic DNA contamination. The purity and concentration were determined by ultraviolet spectrophotometer, and the integrity of RNA was detected by $1.5 \%$ agarose gel electrophoresis. The concentration of RNA was adjusted to $500 \mathrm{ng} / \mu \mathrm{l}$, and RNA samples were reverse transcribed into cDNAs by using reverse transcriptase in strict accordance with the instructions. RT-PCR system was $20 \mu \mathrm{l}$, 2x Ultra SYBR-Green one step Qrt-PCR buffer $10 \mu \mathrm{l}$, RNA template $2 \mu \mathrm{l}$, nuclease-free water $5.5 \mu \mathrm{l}, 1 \mu \mathrm{l}$ of upstream and downstream primers, and super enzyme mix $0.5 \mu \mathrm{l}$. RT-PCR reaction conditions were: Pre-denaturation at $95^{\circ} \mathrm{C}$ for $10 \mathrm{~min}$, denaturation at $95^{\circ} \mathrm{C}$ for $15 \mathrm{sec}$, annealing, and extension at $60^{\circ} \mathrm{C}$ for $1 \mathrm{~min}$, for a total of 40 cycles. The primers in this experiment were designed by Primer Premier 5.0 (Premier Biosoft International) primer design software, and generated by Tianjin Sell Biotechnology Co., Ltd. U6 was used as an internal reference. The specific primer sequences are shown in Table I. The above system configuration was strictly in accordance with the instructions. In the results, the number of cycles of the fluorescent signal cycle $\mathrm{Ct}$ is the number of cycles corresponding to the inflection point at which the background begins to enter the exponential growth phase during the amplification process. The relative expression levels of the target gene miR-129-5p and miR-433 were calculated by $2^{-\Delta C t}$. 
Table I. Primer sequences.

\begin{tabular}{lll}
\hline Genes & \multicolumn{1}{c}{ Upstream primers } & \multicolumn{1}{c}{ Downstream primers } \\
\hline miR-129-5p & 5'-CTTTTTGCGGTCTGGGCTTG-3' & 5'-AACGCTTCACGAATTTGCGT-3' \\
miR-433 & 5'-GGATCATGATGGGCTCCT-3' & 5'-CAGTGCGTGTCGTGGAGT-3' \\
U6 & 5'-CTCGCTTCGGCAGCACA-3' & 5'-AACGCTTCACGAATTTGC-3'
\end{tabular}

Table II. Comparison of general clinical data between the two groups [mean $\pm \mathrm{SD}, \mathrm{n}(\%)]$.

\begin{tabular}{|c|c|c|c|c|}
\hline Variables & Research group $(\mathrm{n}=78)$ & Control group $(n=72)$ & $t / \chi^{2}$ & P-value \\
\hline Age (years) & $58.52 \pm 7.96$ & $57.98 \pm 8.57$ & 0.40 & 0.69 \\
\hline BMI $\left(\mathrm{kg} / \mathrm{m}^{2}\right)$ & $20.22 \pm 2.08$ & $20.12 \pm 2.11$ & 0.29 & 0.77 \\
\hline Smoking & & & 0.05 & 0.83 \\
\hline Yes & $24(30.77)$ & $21(29.16)$ & & \\
\hline No & $54(69.23)$ & $51(70.84)$ & & \\
\hline Drinking & & & 0.01 & 0.94 \\
\hline Yes & $21(26.92)$ & $19(26.39)$ & & \\
\hline No & $57(73.08)$ & $53(73.61)$ & & \\
\hline Diastolic pressure $(\mathrm{mmHg})$ & $77.23 \pm 11.13$ & $77.56 \pm 11.45$ & 0.18 & 0.86 \\
\hline Systolic pressure (mmHg) & $112.34 \pm 15.24$ & $111.19 \pm 16.15$ & 0.45 & 0.65 \\
\hline WBC $\left(\times 10^{9} / 1\right)$ & $5.59 \pm 4.35$ & $5.71 \pm 3.91$ & 0.18 & 0.86 \\
\hline $\mathrm{HB}(\mathrm{gm} / \mathrm{dl})$ & $12.17 \pm 2.11$ & $11.69 \pm 1.96$ & 1.44 & 0.15 \\
\hline $\operatorname{PLT}\left(\times 10^{9} / 1\right)$ & $153.97 \pm 20.91$ & $154.27 \pm 21.61$ & 0.09 & 0.93 \\
\hline $\operatorname{RBC}(1,012 / 1)$ & $4.56 \pm 0.41$ & $4.64 \pm 0.38$ & 1.24 & 0.22 \\
\hline
\end{tabular}

SD, standard deviation; MBI, body mass index; WBC, white blood cells; HB, hemoglobin; PLT, platelet red blood cells; RBC red blood cells.

Observation indicators. The basic clinical data of the two groups were compared. The expression levels of miR-129-5p and miR-433 in peripheral blood of the research group and the control group were observed. The relationship between the expression of miR-129-5p, miR-433 and clinicopathological features was analyzed. The association between the expression levels of miR-129-5p, miR-433, the clinical stage, and differentiation of breast cancer was analyzed.

Statistical analysis. The experimental data were analyzed by SPSS 19.0 software system (IBM Corp.). The counting data were expressed as n (\%), and Chi-square test was used for comparison between groups. The measured data were expressed as (mean $\pm \mathrm{SD}$ ). Paired t-test was used to compare the two groups. One-way ANOVA was used to compare the mean of multiple groups. LSD t-test was used for post-test. Spearman's correlation coefficient was used to evaluate the correlation between the expression levels of miR-129-5p, miR-433, clinical stages and degree of differentiation. The sensitivity and specificity of single and combined detection were evaluated by the receiver operating curve (ROC). The diagnostic value of combined detection of miR-129-5p and miR-433 in breast cancer was analyzed by binary logistic regression. $\mathrm{P}<0.05$ was considered statistically significant.

\section{Results}

Comparison of general data. General clinical data of age, body mass index, smoking and drinking, diastolic blood pressure, systolic blood pressure, white blood cell (WBC), hemoglobin (HB), red blood cell (RBC) count and platelet (PLT) count were compared between the research and the control group. There was no difference in general clinical data between the two groups $(\mathrm{P}>0.05)$ (Table II).

Comparison of the expression levels of miR-129-5p and miR-433 in two groups. The expression levels of miR-129-5p and miR-433 in the two groups were detected by RT-PCR (Fig. 1). The expression level of miR-129-5p in the research group $(0.84 \pm 0.45)$ was significantly lower than that in the control group $(1.58 \pm 0.89)(\mathrm{P}<0.05)$. The expression level of miR-433 in the research group $(0.59 \pm 0.32)$ was significantly lower than that in the control group $(1.68 \pm 0.87)(\mathrm{P}<0.05)$.

Relationship between the expression levels of miR-129-5p, miR-433 and clinicopathological features in breast cancer patients. Clinical information of the research group (Table III), indicated that the expression level of miR-129-5p in the blood of breast cancer patients was not significantly correlated with 
Table III. Relationship between the expression levels of miR-129-5p, miR-433 and clinicopathological features (mean \pm SD).

\begin{tabular}{|c|c|c|c|c|c|c|c|}
\hline Variables & Cases & miR-129-5p & $\mathrm{F} / \mathrm{t}$ & P-value & $\operatorname{miR}-433$ & $\mathrm{~F} / \mathrm{t}$ & P-value \\
\hline Age, years & & & 0.27 & 0.78 & & 0.40 & 0.69 \\
\hline$<50$ & 42 & $0.88 \pm 0.49$ & & & $0.57 \pm 0.21$ & & \\
\hline$\geq 50$ & 36 & $0.91 \pm 0.47$ & & & $0.59 \pm 0.23$ & & \\
\hline Tumor size, $\mathrm{cm}$ & & & 2.78 & 0.01 & & 1.01 & 0.31 \\
\hline$<5$ & 56 & $1.12 \pm 0.44$ & & & $0.54 \pm 0.20$ & & \\
\hline$\geq 5$ & 22 & $0.82 \pm 0.40$ & & & $0.49 \pm 0.19$ & & \\
\hline Differentiation & & & 53.47 & $<0.01$ & & 41.28 & $<0.01$ \\
\hline High differentiation & 16 & $1.23 \pm 0.36$ & & & $0.91 \pm 0.24$ & & \\
\hline Medium differentiation & 45 & $0.93 \pm 0.11$ & & & $0.52 \pm 0.21$ & & \\
\hline Low differentiation & 17 & $0.55 \pm 0.12$ & & & $0.29 \pm 0.10$ & & \\
\hline Lymph node metastasis & & & 5.45 & $<0.01$ & & 5.38 & $<0.01$ \\
\hline Yes & 48 & $0.65 \pm 0.34$ & & & $0.38 \pm 0.24$ & & \\
\hline No & 30 & $1.18 \pm 0.52$ & & & $0.69 \pm 0.26$ & & \\
\hline Infiltration depth & & & 6.28 & $<0.01$ & & 3.82 & $<0.01$ \\
\hline $\mathrm{T} 1+\mathrm{T} 2$ & 28 & $1.08 \pm 0.33$ & & & $0.72 \pm 0.27$ & & \\
\hline $\mathrm{T} 3+\mathrm{T} 4$ & 50 & $0.61 \pm 0.31$ & & & $0.51 \pm 0.21$ & & \\
\hline Clinical stages & & & 33.42 & $<0.01$ & & 79.50 & $<0.01$ \\
\hline I & 16 & $1.28 \pm 0.43$ & & & $0.91 \pm 0.11$ & & \\
\hline II & 25 & $0.82 \pm 0.31$ & & & $0.71 \pm 0.16$ & & \\
\hline III & 21 & $0.54 \pm 0.11$ & & & $0.42 \pm 0.13$ & & \\
\hline IV & 16 & $0.40 \pm 0.12$ & & & $0.27 \pm 0.11$ & & \\
\hline
\end{tabular}
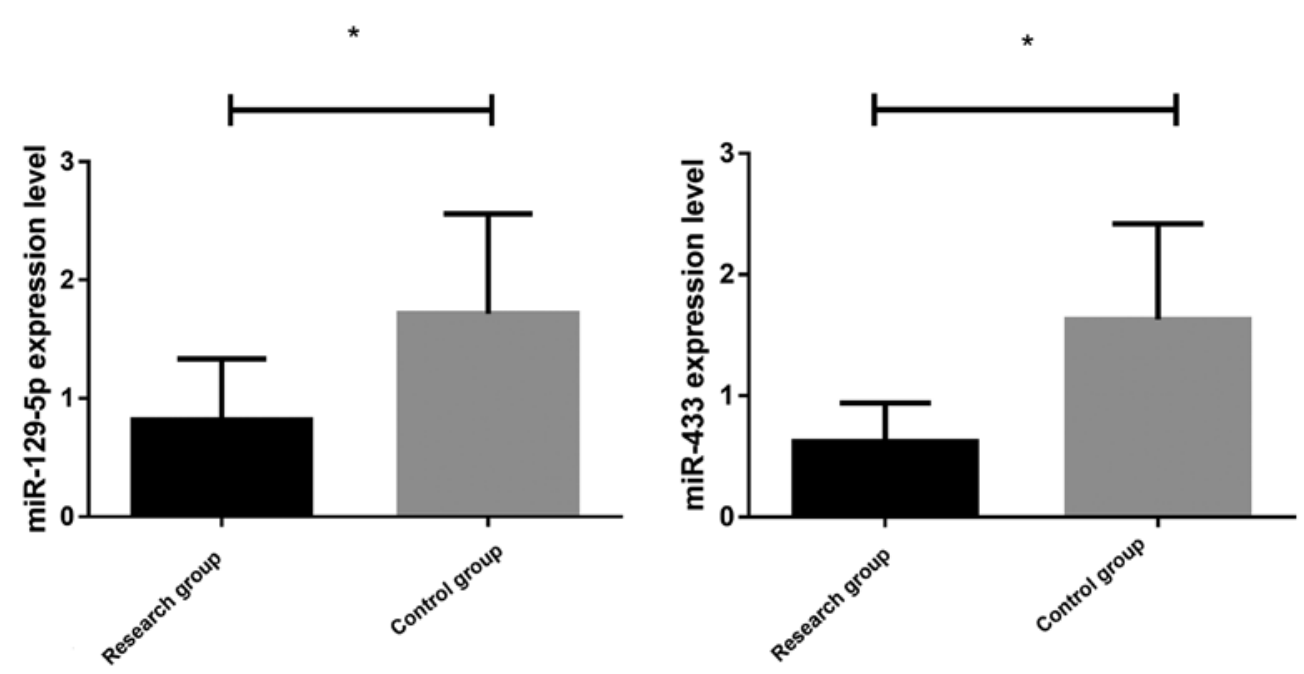

Figure 1. Comparison of expression levels of miR-129-5p and miR-433 in two groups. The expression levels of miR-129-5p and miR-433 in the research group were significantly lower than those in the control group, with significant differences. ${ }^{*} \mathrm{P}<0.05$ vs. the two groups.

age $(\mathrm{P}>0.05)$, but it was correlated with tumor size, differentiation degree, lymph node metastasis, invasion depth and clinical stage $(\mathrm{P}<0.05)$. The expression level of miR-433 was not significantly correlated with age or tumor size $(\mathrm{P}>0.05)$, but was correlated with differentiation, lymph node metastasis, depth of invasion and clinical stage $\mathrm{P}<0.05$ ).

Correlation between the expression level of $\mathrm{miR}-129-5 \mathrm{p}$, miR-433, clinical stage and the degree of differentiation. Expression levels of miR-129-5p and miR-433 in the two groups were detected by RT-PCR, and the correlation between the expression levels of miR-129-5p, miR-433 and the clinical stage of breast cancer was analyzed (Fig. 2). The expression of miR-129-5p and miR-433 was negatively correlated with clinical stages $(r=-0.6595,-0.8947 ; \mathrm{P}<0.05)$. The expression levels of miR-129-5p and miR-433 decreased gradually with the aggravation of patient's condition.

Correlation between the expression level of miR-129-5p, miR-433 and the degree of differentiation. The expression 

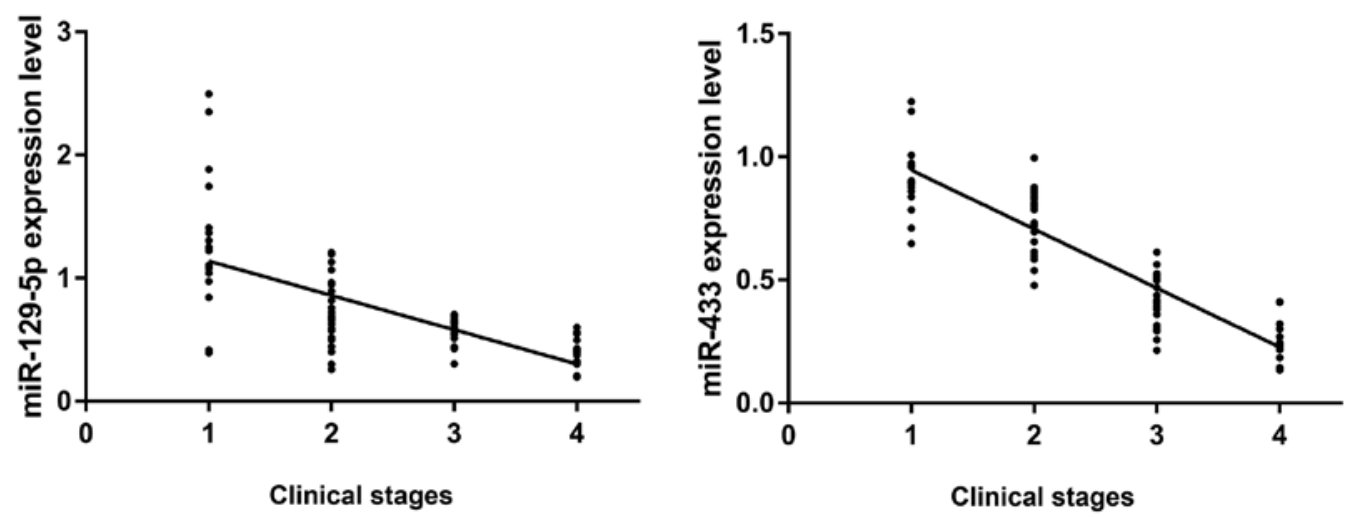

Figure 2. Correlation between expression levels of miR-129-5p, miR-433 and clinical stages. There was a significant negative correlation between miR-129-5p, miR-433 and clinical stages. As the disease aggravated (clinical stages increased), the expression levels of miR-129-5p and miR-433 gradually decreased. Clinical stage 1: Phase I; stage 2: Phase II; stage 3: Phase III; stage IV: Phase IV.
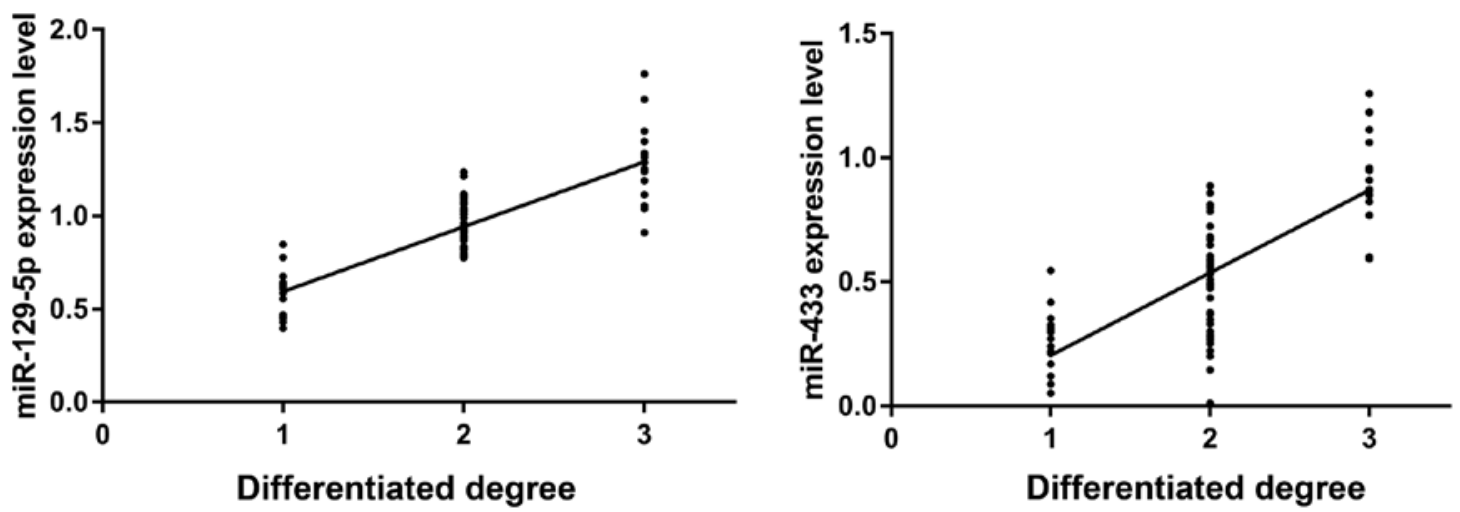

Figure 3. Correlation between the expression levels of miR-129-5p, miR-433 and the degree of differentiation. miR-129-5p and miR-433 were significantly positively correlated with the degree of differentiation. The higher the degree of differentiation, the higher the expression levels of miR-129-5p and miR-433. Degree of differentiation 1: Poor differentiation; 2: Moderate differentiation; 3: High differentiation.

levels of miR-129-5p and miR-433 were detected by RT-PCR. Correlation between the expression levels of miR-129-5p, miR-433 and the differentiation of breast cancer cells was analyzed (Fig. 3). There was a significant positive correlation between the expression of miR-129-5p, miR-433 and the differentiation of cancer cells $(r=0.8507, r=0.7522 ; \mathrm{P}<0.05)$. The higher the differentiation of cancer cells, the higher the expression levels of miR-129-5p and miR-433.

Comparison of the value of $\mathrm{miR}-129-5 \mathrm{p}$ and $\mathrm{miR}-433$ in the diagnosis of breast cancer by single and combined detection. Sensitivity, specificity and AUC of single and combined detection of miR-129-5p and miR-433 were compared between the research group and the control group (Table IV). High to low sensitivity was combined, detection rate $(87.5 \%)$, miR-433 (73.61\%) and miR-129-5p (69.44\%). The specificity from high to low was miR-433 (93.59\%), combined detection $(89.74 \%)$ and miR-129-5p (83.33\%). The highest level of AUC combined detection was 0.95 (Fig. 4).

Association between miR-129-5p, miR-433 and prognosis of breast cancer patients. Patients were followed up for 1 year, and no patients were lost. The results showed that the survival rate of breast cancer was $80.77 \%$. According to the median values of the expression levels of miR-129-5p and miR-433, patients were divided into high-expression groups and low expression groups, and the survival curve was plotted. miR-129-5p low expression group $(n=39)$ had lower survival than the high expression group $(n=39)$, and the miR-433 low expression group $(n=39)$ had lower survival than the high expression group $(\mathrm{n}=39)(\mathrm{P}<0.05)$ (Fig. 5).

\section{Discussion}

Breast cancer patients often have poor prognosis due to early noninvasive diagnosis, drug resistance and metastasis in the treatment of breast cancer (26). Therefore, it is worth exploring the ideal molecular targets for early diagnosis and targeted therapy of breast cancer $(27,28)$. MicroRNAs play a very important regulatory role in the early diagnosis, clinical stages and prognosis, and in reversing drug resistance of tumors (29). miR-129-5p was discovered as a tumor-suppressing microRNA. In the study of Luan et al (30), miR-129-5p expression was low in breast cancer and regulated multidrug resistance (MDR) in breast cancer cells. In the present study, the expression level of miR-129-5p in the research group was significantly lower than that in the control group $(\mathrm{P}<0.05)$. The expression level of miR-129-5p in the blood of breast cancer patients was positively correlated with tumor size, degree of differentiation, lymph node metastasis, invasion depth and 
Table IV. Comparison of the diagnostic value of single and combined detection of miR-129-5p and miR-433 in breast cancer.

\begin{tabular}{lcccccccc}
\hline & \multicolumn{9}{c}{$\begin{array}{c}\text { Sensitivity } \\
\text { Detection }\end{array}$} & $\begin{array}{c}\text { Specificity } \\
(\%)\end{array}$ & $\begin{array}{c}\text { Youden } \\
\text { index }\end{array}$ & $\begin{array}{c}\text { Optimum } \\
\text { critical } \\
\text { value }\end{array}$ & AUC & P-value & Upper limit & Lower limit \\
\hline miR-129-5p & 69.44 & 83.33 & 0.03 & $>1.30$ & 0.83 & $<0.01$ & 0.89 & 0.76 \\
miR-433 & 73.61 & 93.59 & 0.03 & $>1.14$ & 0.88 & $<0.01$ & 0.94 & 0.82 \\
miR-129-5p+miR-433 & 87.5 & 89.74 & 0.02 & $>0.46$ & 0.95 & $<0.01$ & 0.99 & 0.91 \\
\hline
\end{tabular}

CI, confidence intervals.
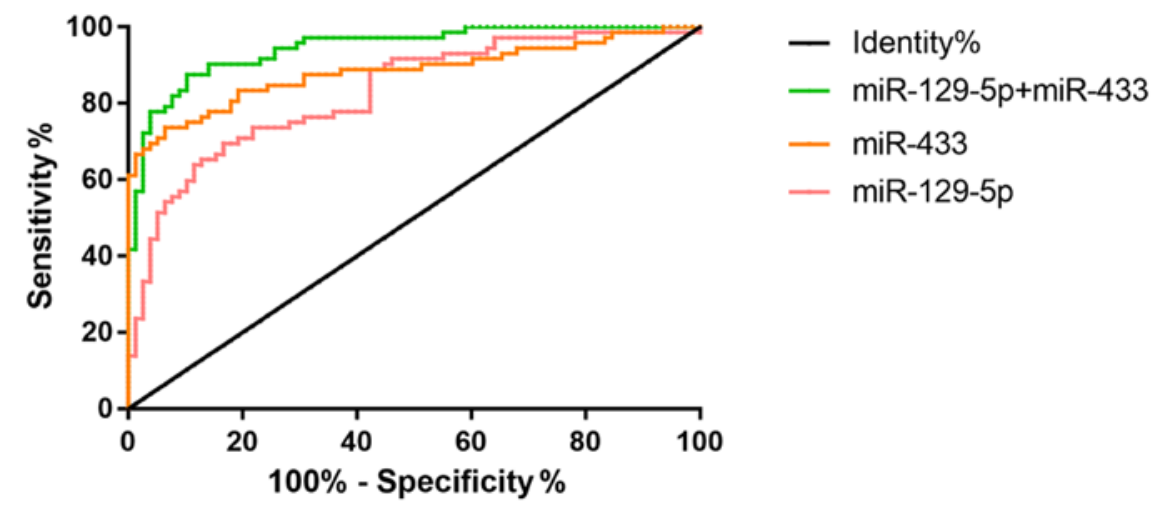

Figure 4. ROC curves were drawn by miR-129-5p and miR-433 with single and joint detection. The single and joint detection of area under the ROC curve of miR-129-5p and miR-433, showing joint detection was the highest at 0.95 .

A

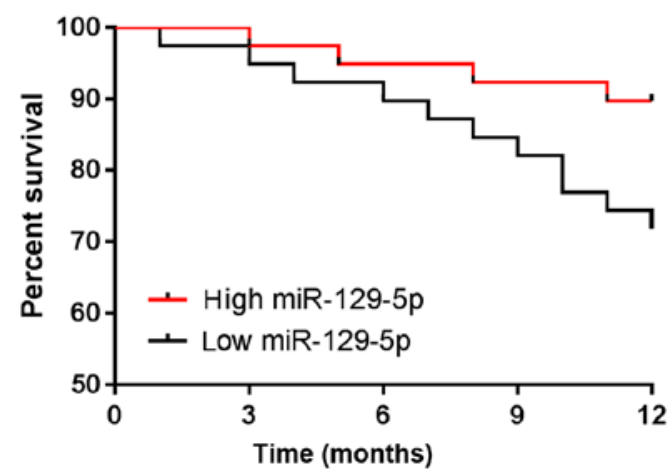

B

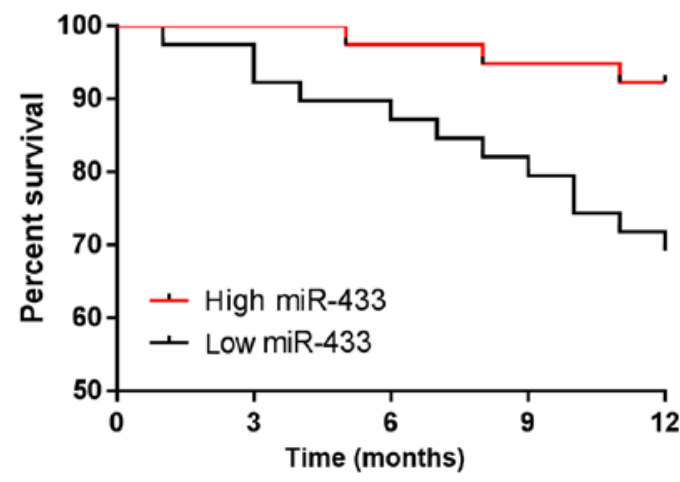

Figure 5. Association between miR-129-5p, miR-433 and prognosis of breast cancer patients. (A) One-year survival of patients with high and low expression of miR-129-5p. (B) One-year survival of patients with high and low expression of miR-433.

clinical stage $(\mathrm{P}<0.05)$. It is positively correlated with the degree of differentiation. The higher the degree of differentiation, the higher the expression level, indicating that miR-129-5p plays an important role in the occurrence and progression of breast cancer. Moreover, it is negatively correlated with clinical stage. The lower the expression level, the higher the severity of the disease, indicating that miR-129-5p can be used to judge the severity of the disease. In the study by Luo et al (31), HMGB1 is shown as direct functional target of miR-129-5p in breast cancer cells. miR-129-5p may inhibit autophagy of breast cancer cells by targeting HMGB-1, and may also reduce the radiation resistance of breast cancer cells. It has also been shown that targeting inhibition of HMGB1 expression by miR-129-5p inhibits the proliferation and migration of osteosarcoma cells (32) and enhance the sensitivity of breast cancer MCF-7 and MDA-MB-231 cells to radiotherapy (33). The authors speculate that miR-129-5p can promote breast cancer cell apoptosis by targeting inhibition of HMGB1 or similar nuclear proteins, and may also be an important way to regulate the radiosensitivity of breast cancer cells. The expression of miR-433 in the research group was significantly lower than that in the control group $(\mathrm{P}<0.05)$. The expression of miR-433 was correlated with the degree of tumor differentiation, lymph node metastasis, depth of invasion and clinical stage $(\mathrm{P}<0.05)$. There 
is a positive correlation with the degree of differentiation. The higher the degree of differentiation, the higher the expression level indicating that miR-433 also plays an important role in the occurrence and progression of breast cancer. There was a significant negative correlation with clinical stage. As the condition becomes more serious, the expression level declines, indicating that miR-433 can be used to judge the severity of patient's condition. Gotanda et al (34) and Wang et al (35) studied apoptosis of retinoblastoma and human dental pulp cells induced by miR-433. Zhang et al (36) revealed the effect of miR-433 on apoptosis, migration and proliferation of breast cancer cells. The results of the above studies confirmed that Rapla, as a direct target gene of miR-433, participates in the function of miR-433. Rapla activated the MAPK signaling pathway, promoting cell migration, proliferation and inhibiting apoptosis. These findings highlight that miR-433 is an anti-oncogene that regulates the progression and metastasis potential of breast cancer and may contribute to the future development of treatment for breast cancer. Referring to the relevant literature, there are few studies on the diagnosis of breast cancer by miR-129-5p and miR-433, and no studies on the combined diagnosis of the two miRs. In this study, the diagnostic significance of miR-129-5p and miR-433 with single and combined detection in breast cancer is compared, and it was found that the sensitivity and specificity of miR-129-5p with single detection were 73.61 and $83.33 \%$, respectively. The sensitivity and specificity with single detection of miR-433 were 73.61 and $93.59 \%$, respectively. The sensitivity and specificity in the combined detection of miR-129-5p and miR-433 were 87.5 and $89.74 \%$, respectively. The optimal thresholds of miR-129-5p and miR-433 were 1.30 and 1.14 , respectively, and the diagnostic efficiency was the highest at this point. The results suggest that the combined detection of miR-129-5p and miR-433 is the most sensitive. The value of the combined detection of miR-129-5p and miR-433 in the diagnosis of breast cancer was evaluated. ROC curves were drawn based on the sensitivity and specificity with the single and combined detection. The larger the AUC, the greater the diagnostic value. The combined diagnosis AUC (0.95) is larger than the single diagnosis AUC $(0.83,0.88)$ (Fig. 4). The above indicates that the value of the combined detection of miR-129-5p and miR-433 is higher than the value of its single detection. It has been reported that low level of miR-129-3p is associated with shortterm disease-free survival and overall survival in patients with renal cell carcinoma (37). Moreover, Zheng et al (38) proposed that down-regulation of miR-433 level may be related to poor prognosis of patients with gastrointestinal cancer. Therefore, the one-year survival of breast cancer patients was analyzed in this study. The results showed that the low expression groups of miR-129-5p and miR-433 had lower survival than the high expression groups, suggesting that the poor prognosis of breast cancer patients may be related to the low expression of miR-129-5p and miR-433.

In the present study, the expression levels of miR-129-5p and miR-433 in the blood of patients were studied in many aspects, which provided a reference for clinical research. There were some limitations in this study. In recent years, there have been many studies on serum diagnostic indicators since they are convenient and easy to obtain. Therefore, this study mainly focused on serology to explore its value. Due to the short follow-up time, coupled with the differences in the treatment, the relationship between miR-129-5p and miR-433 and the long-term survival prognosis of breast cancer patients needs to be further studied.

In conclusion, the expression levels of miR-129-5p and miR-433 in peripheral blood of patients with breast cancer are lower than those of healthy people, and are closely related to the clinical stage of breast cancer and the degree of differentiation of cancer cells. It is expected to provide reference for judging the condition of breast cancer patients and to guide the treatment of breast cancer. The combined detection of miR-129-5p and miR-433 is of great significance for the diagnosis and treatment of breast cancer, and will provide a new entry point for targeted treatment of breast cancer.

\section{Acknowledgements}

Not applicable.

\section{Funding}

No funding was received.

\section{Availability of data and materials}

The datasets used and/or analyzed during the present study are available from the corresponding author on reasonable request.

\section{Authors' contributions}

JX conceived the study and wrote the manuscript. XZ and PH conceived and designed the study. $\mathrm{YH}$ and $\mathrm{YX}$ were responsible for the collection and analysis of the experimental data. RL and MZ interpreted the data and drafted the manuscript. JX and $\mathrm{PH}$ revised the manuscript critically for important intellectual content. All authors read and approved the final version of the manuscript.

\section{Ethics approval and consent to participate}

The study was approved by the Ethics Committee of Zhengzhou Central Hospital Affiliated to Zhengzhou University (Zhengzhou, China). Signed informed consents were obtained from the patients and/or guardians.

\section{Patient consent for publication}

Not applicable.

\section{Competing interests}

The authors declare that they have no competing interests.

\section{References}

1. Cronin KA, Lake AJ, Scott S, Sherman RL, Noone AM, Howlader N, Henley SJ, Anderson RN, Firth AU, Ma J, et al: Annual report to the nation on the status of cancer, part I: National cancer statistics. Cancer 124: 2785-2800, 2018.

2. Torre LA, Bray F, Siegel RL, Ferlay J, Lortet-Tieulent J and Jemal A: Global cancer statistics, 2012. CA Cancer J Clin 65: 87-108, 2015. 
3. Yardley DA: Pharmacologic management of bone-related complications and bone metastases in postmenopausal women with hormone receptor-positive breast cancer. Breast Cancer (Dove Med Press) 8: 73-82, 2016.

4. Futakuchi $M$ and Singh RK: Animal model for mammary tumor growth in the bone microenvironment. Breast Cancer 20: 195-203, 2013.

5. Shioi Y, Kashiwaba M, Inaba T, Komatsu H, Sugai $T$ and Wakabayashi G: Long-term complete remission of metastatic breast cancer, induced by a steroidal aromatase inhibitor after failure of a non-steroidal aromatase inhibitor. Am J Case Rep 15: 85-89, 2014.

6. Siegel RL, Miller KD and Jemal A: Cancer Statistics, 2017. CA Cancer J Clin 67: 7-30, 2017.

7. Chen W, Zheng R, Baade PD, Zhang S, Zeng H, Bray F, Jemal A $\mathrm{Yu}$ XQ and He J: Cancer statistics in China 2015. CA Cancer J Clin 66: 115-132, 2016.

8. Fitzmaurice C, Dicker D, Pain A, Hamavid H, Moradi-Lakeh M, MacIntyre MF, Allen C, Hansen G, Woodbrook R, Wolfe C, et al Global burden of disease cancer collaboration: The global burden of cancer 2013. JAMA Oncol 1: 505-527, 2015.

9. Zhang ML, Huang ZZ and Zheng Y: Estimates and prediction on incidence, mortality and prevalence of breast cancer in China, 2008. Zhonghua Liu Xing Bing Xue Za Zhi 33: 1049-1051, 2012 (In Chinese)

10. Chen WQ and Zheng RS: Incidence, morality and survival analysis of breast cancer in China. Chin J Clin Oncol 42: 668-674, 2015 (In Chinese).

11. He K, Li WX, Guan D, Gong M, Ye S, Fang Z, Huang JF and Lu A Regulatory network reconstruction of five essential microRNAs for survival analysis in breast cancer by integrating miRNA and mRNA expression datasets. Funct Integr Genomics 19: 645-658, 2019.

12. Rupaimoole R and Slack FJ: MicroRNA therapeutics: Towards a new era for the management of cancer and other diseases. Nat Rev Drug Discov 16: 203-222, 2017.

13. Agarwal V, Bell GW, Nam JW and Bartel DP: Predicting effective microRNA target sites in mammalian mRNAs. elife, 2015, 4: e05005. doi: 10.7554/eLife.05005.

14. Calin GA, Sevignani C, Dumitru CD, Hyslop T, Noch E, Yendamuri S, Shimizu M, Rattan S, Bullrich F, Negrini M, et al: Human microRNA genes are frequently located at fragile sites and genomic regions involved in cancers. Proc Natl Acad Sci USA 101: 2999-3004, 2004.

15. Ambros V: The functions of animal microRNAs. Nature 431 350-355, 2004

16. Zhang B, Pan X, Cobb GP and Anderson TA: microRNAs as oncogenes and tumor suppressors. Dev Biol 302: 1-12, 2007.

17. Peng X, Cao P, He D, Han S, Zhou J, Tan G, Li W, Yu F, Yu J, Li Z and Cao K: MiR-634 sensitizes nasopharyngeal carcinoma cells to paclitaxel and inhibits cell growth both in vitro and in vivo. Int J Clin Exp Pathol 7: 6784-6791, 2014.

18. Ye J, Zhang Z, Sun L, Fang Y, Xu X and Zhou G: MiR-186 regulates chemo-sensitivity to paclitaxel via targeting MAPT in non-small cell lung cancer (NSCLC). Mol Biosyst 12: 3417-3424, 2016.

19. Duan L, Hao X, Liu Z, Zhang Y and Zhang G: MiR-129-5p is down-regulated and involved in the growth, apoptosis and migration of medullary thyroid carcinoma cells through targeting RET. FEBS Lett 588: 1644-1651, 2016.

20. Jiang Z, Wang H, Li Y, Hou Z, Ma N, Chen W, Zong Z, Chen S MiR-129-5p is down-regulated and involved in migration and invasion of gastric cancer cells by targeting interleukin-8. Neoplasma 63: 673-680, 2016

21. Luan QX, Zhang BG, Li XJ and Guo MY: MiR-129-5p is downregulated in breast cancer cells partly due to promoter $\mathrm{H} 3 \mathrm{~K} 27 \mathrm{~m} 3$ modification and regulates epithelial-mesenchymal transition and multi-drug resistance. Eur Rev Med Pharmacol Sci 20: 4257-4265, 2016.
22. Luo H, Zhang H, Zhang Z, Zhang X, Ning B, Guo J, Nie N, Liu B and Wu X: Down-regulated miR-9 and miR-433 in human gastric carcinoma. J Exp Clin Cancer Res 28: 82, 2009.

23. Tan Y, Ge G, Pan T, Wen D, Chen L, Yu X, Zhou X and Gan J: A serum microRNA panel as potential biomarkers for hepatocellular carcinoma related with hepatitis B virus. PLoS One 9: e107986, 2014.

24. Zhang T, Jiang K, Zhu X, Zhao G, Wu H, Deng G and Qiu C: miR-433 inhibits breast cancer cell growth via the MAPK signaling pathway by targeting Rapla. Int J Biol Sci 14: 622-632, 2018.

25. Meng R, Fang J, Yu Y, Hou LK, Chi JR, Chen AX, Zhao Y and Cao XC: miR-129-5p suppresses breast cancer proliferation by targeting CBX4. Neoplasma 65: 572-578, 2018.

26. Zdenkowski N, Butow P, Tesson S and Boyle F: A systematic review of decision aids for patients making a decision about treatment for early breast cancer. Breast 26: 31-45, 2016.

27. Wu X, Zeng R, Wu S, Zhong J, Yang L and Xu J: Comprehensive expression analysis of miRNA in breast cancer at the miRNA and isomiR levels. Gene 557: 195-200, 2015.

28. D'Angelo B, Benedetti E, Cimini A and Giordano A: MicroRNAs: A puzzling tool in cancer diagnostics and therapy. Anticancer Res 36: 5571-5575, 2016

29. Yan JW, Lin JS and He XX: The emerging role of miR-375 in cancer. Int J Cancer 135: 1011-1018, 2014.

30. Luan QX, Zhang BG, Li XJ and Guo MY: MiR-129-5p is downregulated in breast cancer cells partly due to promoter $\mathrm{H} 3 \mathrm{~K} 27 \mathrm{~m} 3$ modification and regulates epithelial-mesenchymal transition and multi-drug resistance. Eur Rev Med Pharmacol Sci 20: 4,257-4,265, 2016 .

31. Luo J, Chen J and He L: miR-129-5p attenuates irradiationinduced autophagy and secreases radioresistance of breast cancer cells by targeting HMGB1. Med Sci Monit 21: 4122-4129, 2015.

32. Liu K, Huang J, Ni J, Song D, Ding M, Wang J, Huang X and Li W: MALAT1 promotes osteosarcoma development by regulation of HMGB1 via miR-142-3p and miR-129-5p. Cell Cycle 16: 578-587, 2017.

33. Luo J, Chen J and He L: Mir-129-5p attenuates irradiation-induced autophagy and decreases radioresistance of breast cancer cells by targeting HMGB1. Med Sci Monit 21: 4122-4129, 2015

34. Gotanda K, Hirota T, Matsumoto N and Ieiri I: MicroRNA-433 negatively regulates the expression of thymidylate synthase (TYMS) responsible for 5-fluorouracil sensitivity in HeLa cells. BMC Cancer 13: 369, 2013.

35. Wang K, Li L, Wu J, Qiu Q, Zhou F and Wu H: The different expression profiles of microRNAs in elderly and young human dental pulp and the role of miR-433 in human dental pulp cells. Mech Ageing Dev 146-148: 1-11, 2015.

36. Zhang T, Jiang K, Zhu X, Zhao G, Wu H, Deng G and Qiu C: miR-433 inhibits breast cancer cell growth via the MAPK signaling pathway by targeting Rapla. Int J Biol Sci 14: 622-632, 2018.

37. Chen X, Ruan A, Wang X, Han W, Wang R, Lou N, Ruan H, Qiu B, Yang H and Zhang X: miR-129-3p, as a diagnostic and prognostic biomarker for renal cell carcinoma, attenuates cell migration and invasion via downregulating multiple metastasisrelated genes. J Cancer Res Clin Oncol 140: 1295-1304, 2014.

38. Zheng Q, Chen C, Guan H, Kang W and Yu C: Prognostic role of microRNAs in human gastrointestinal cancer: A systematic review and meta-analysis. Oncotarget 8: 46611-46623, 2017.

(i) 1 This work is licensed under a Creative Commons Attribution-NonCommercial-NoDerivatives 4.0 International (CC BY-NC-ND 4.0) License. 